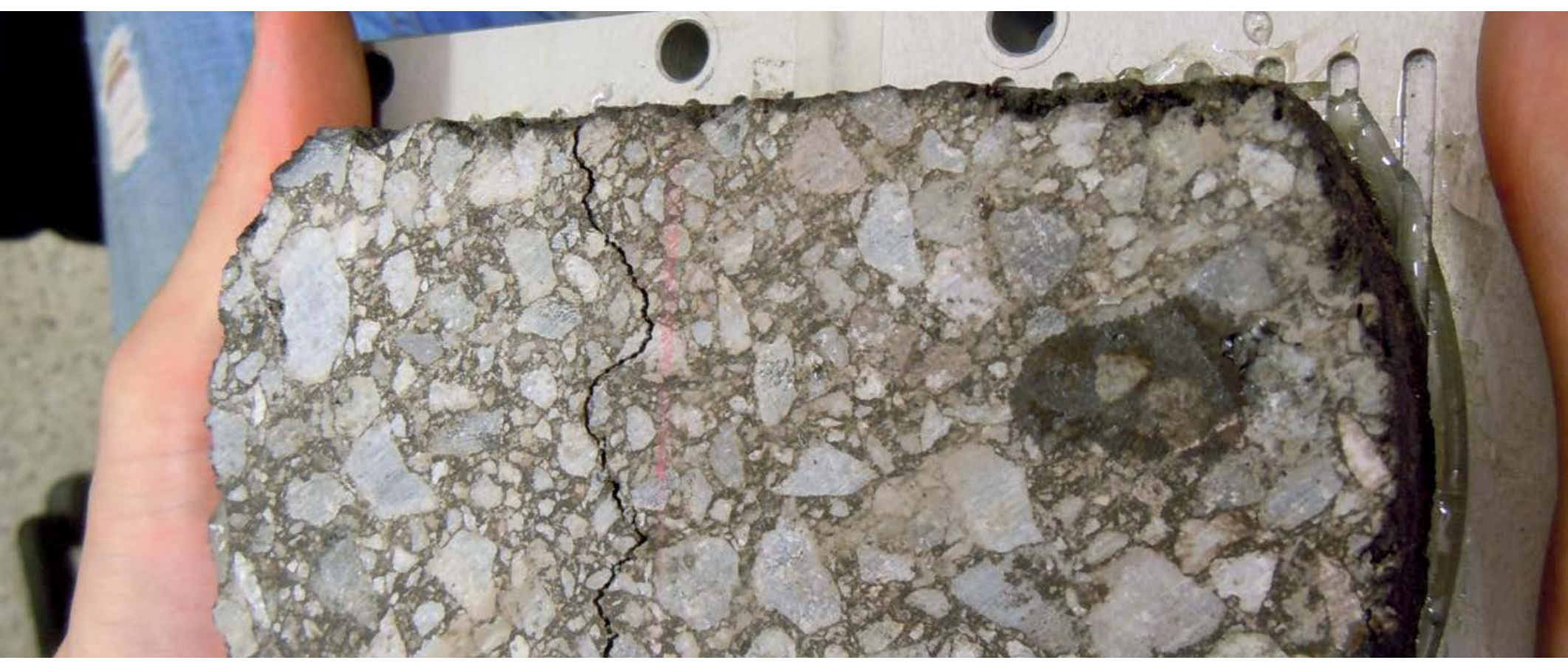

\title{
Overlay Tester: equipo de laboratorio para evaluar la resistencia al agrietamiento en mezclas asfálticas
}

Fecha de recepción: 11 de setiembre de 2013

Fecha de aceptación: 08 de octubre de 2013

\section{Resumen}

La resistencia al agrietamiento debe ser un tema a tomar en cuenta en el desempeño de la mezcla asfáltica, la cual tiene que resistir la deformación permanente, fatiga y el daño por humedad. Las grietas aparecen en los pavimentos flexibles a través de dos mecanismos de falla: fatiga y reflejo de grietas.

Las grietas por reflejo son un tipo de falla que ha recibido menos atención que el agrietamiento por fatiga. El agrietamiento por reflejo debe ser tomado en cuenta cuando se selecciona la mezcla asfáltica como sobrecapa a colocar sobre pavimentos rígidos, bases estabilizadas o simplemente cuando la capa existente está muy agrietada. Es bien sabido que las grietas existentes se propagan a la capa superior provocando que el agua se infiltre causando un rápido deterioro de la estructura del pavimento.

Este artículo se enfoca en la implementación del ensayo de overlay, el cual es un ensayo muy práctico que da suficiente información de las características de agrietamiento de las mezclas asfálticas desde la formación de la grieta hasta su comportamiento de propagación, además es lo suficientemente sensible a los cambios en los contenidos y tipo de asfalto para discriminar entre distintos tipos de mezclas.

Palabras clave: agrietamiento, fisuras, ensayo de overlay, fatiga.
Ing. Mónica Jiménez Acuña,

Jefe, Laboratorio de Ensayos Dinámicos para Pavimentos, LanammeUCR, Universidad de Costa Rica

Tel.: (506) 2511-2513 / E-mail: monica.jimenez@ucr.ac.cr

\section{Abstract}

The crack resistance should be an issue to take into account in the performance of the asphalt mixture, which has to resist permanent deformation, fatigue and moisture damage. Cracks appear in flexible pavements through two failure mechanisms: reflective cracking and fatigue.

The reflective cracking is a type of failure that has received less attention than fatigue cracking. This form of distress should be consider when selecting the asphalt mixture as overlay, to be placed on rigid pavements stabilized bases or simply when the existing layer is very cracked. It is well known that existing cracks propagate to the top layer causing the water to infiltrate causing the rapid deterioration of the pavement structure.

This article focuses on the implementation of the overlay test, which is a very practical method which gives enough information about the characteristic cracking of asphalt mixtures, from the beginning of the crack until its propagation behavior, it's also sensitive enough to measure the changes in the type and asphalt content to discriminate between various types of asphalt mixtures.

Key Words: fatigue cracking, cracks, overlay test. 


\section{Introducción}

En Costa Rica ya existen especificaciones para asegurar el desempeño de la mezcla asfáltica, que minimizan la ocurrencia de fallas por deformación permanente y fisuramiento por fatiga. El uso de asfaltos más rígidos así como de materiales reciclados RAP-RAS ha provocado la reducción de la deformación permanente pero ha hecho que las mezclas sean menos flexibles, por lo tanto, menos resistentes al agrietamiento.

La resistencia al agrietamiento debe ser un tema a tomar en cuenta en el desempeño de la mezcla asfáltica, la cual tiene que resistir la deformación permanente, fatiga y el daño por humedad. Las grietas aparecen en los pavimentos flexibles a través de dos mecanismos de falla: fatiga y reflejo de grietas.

Las fisuras por fatiga clásicas están asociadas a las áreas débiles en la estructura del pavimento donde las cargas pesadas de tránsito inducen mayores deformaciones a tensión en la fibra inferior de la capa de mezcla asfáltica (figura 1a). Estas deformaciones inician la fisura que eventualmente se propaga a la superficie. La propiedad de fatiga se ha estudiado intensivamente durante 40 años que ha resultado en un mejor entendimiento de este mecanismo de falla así como en el desarrollo de equipos de laboratorio como la viga de fatiga a flexotracción. Sin embargo, el ensayo de fatiga se usa como ítem de aceptación pues es un ensayo que toma mucho tiempo, puede requerirse de varias semanas para obtener una sola curva de fatiga [Ref. 3].

Las grietas por reflejo, por otro lado, se inician por la existencia de defectos en las capas inferiores tales como: grietas longitudinales (figura 1b), juntas en pavimentos rígidos o semirrígidos (figura 1c) y áreas con daño por humedad (figura 1d). La causa de este agrietamiento puede estar asociada a efectos del movimiento de apertura y cerradura de las grietas inducidas por las variaciones de temperatura diarias y cargas de tránsito.

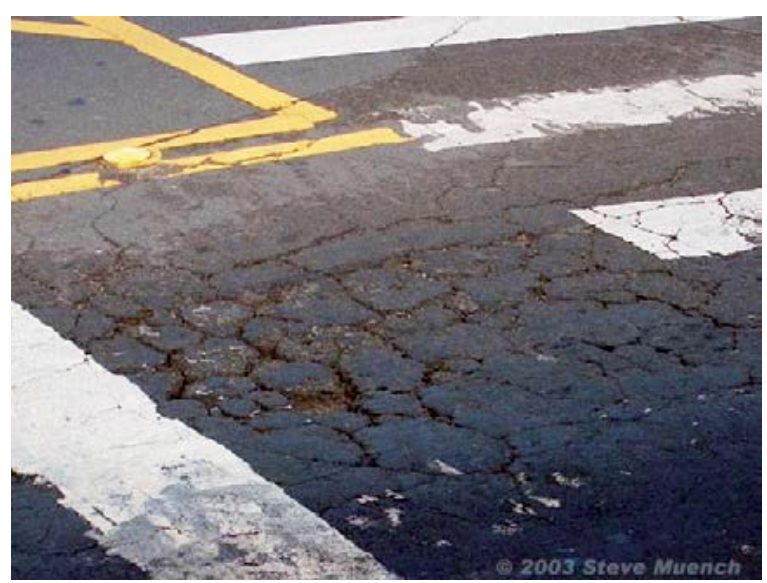

a. Fatiga en la huella de la llanta http://www.stmuench.com/modules/03_general_guidance/03_ pavement_distress.htm

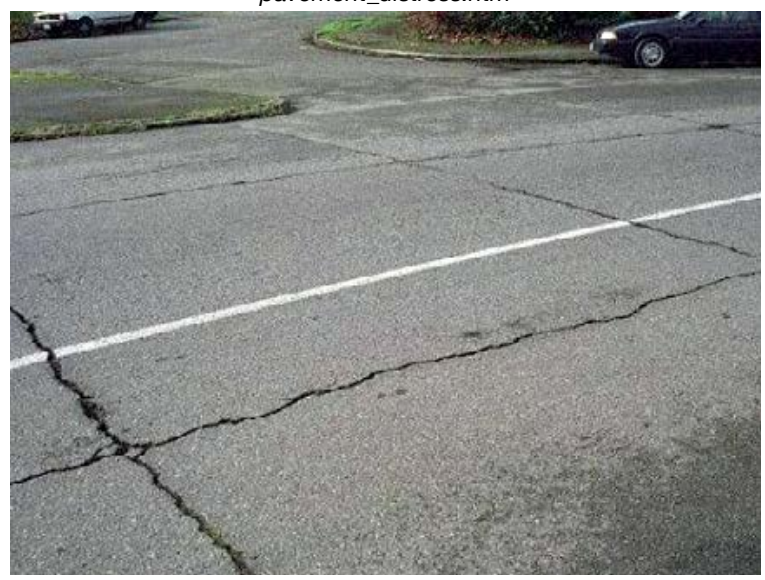

c. Juntas pavimento rígido http://www.roadscience.net/services/distress-guide

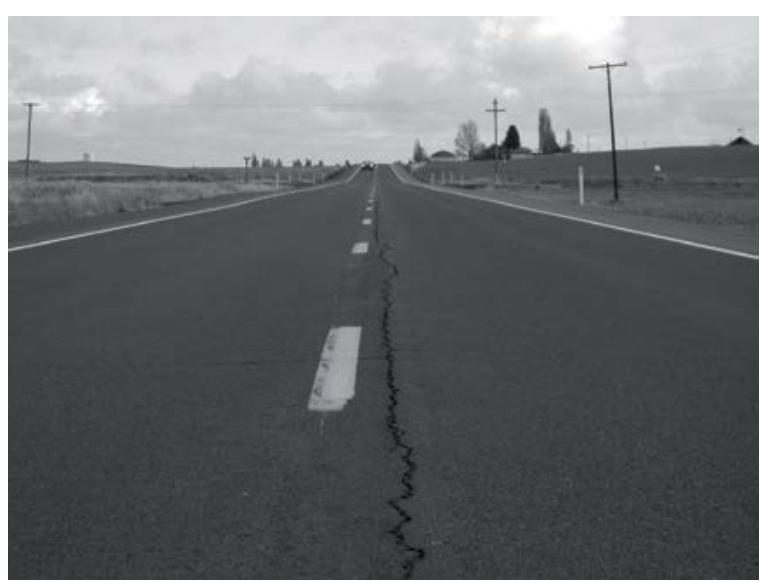

b. Grieta longitudinal

http://sableasphalt.com/asphalt-2/a-crack-is-a-pothole-waiting-to-

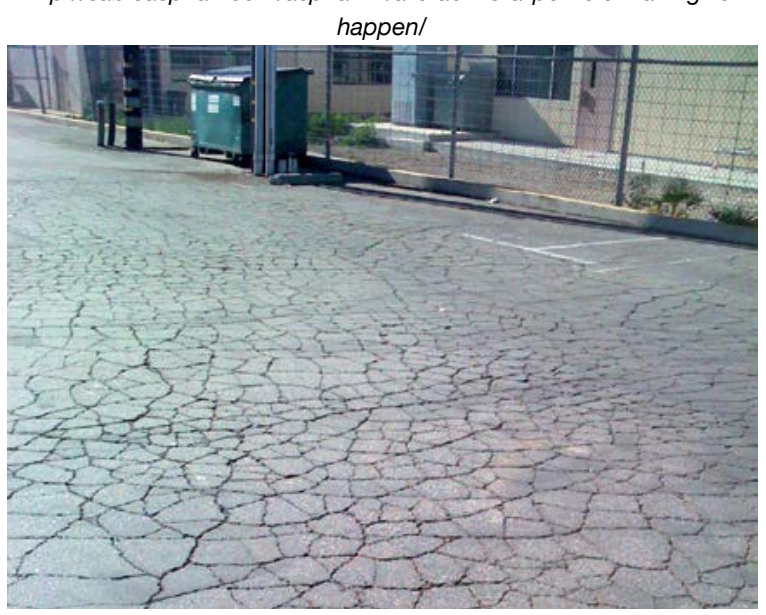

d. Grietas daño por humedad

http://centurypaving.wordpress.com/tag/asphalt-cracks-2/

Figura 1. Tipos de falla por agrietamiento

Fuente: Varios, 2013 
Este tipo de falla ha recibido menos atención que el agrietamiento por fatiga. El agrietamiento por reflejo debe ser un tema a tomar en cuenta cuando se selecciona la mezcla asfáltica como sobrecapa a colocar sobre pavimentos rígidos, bases estabilizadas o simplemente cuando la capa existente está muy agrietada. Es bien sabido que las grietas existentes se propagan a la capa superior provocando que el agua se infiltre causando un rápido deterioro de la estructura del pavimento [Ref. 3].

Todavía no se ha desarrollado un equipo y procedimiento de ensayo simple y rápido de uso rutinario para caracterizar la resistencia al agrietamiento de mezclas asfálticas antes de ser colocadas en un pavimento ya fisurado.

En el mundo existen varios ensayos relacionados con agrietamiento como: ensayo de tracción indirecta (IDT por sus siglas en inglés), ensayo de flexión semicircular (SCB), ensayo de tensión directa (DT), ensayo de tensión directa en forma de disco compacto (DSCTT) y el ensayo de overlay (OV). Todos estos ensayos son rápidos y simples de aplicar, pero ninguno ha podido ser validado con resultados de campo con excepción del ensayo de overlay. Es por esta razón que este artículo se enfoca en la implementación del ensayo de overlay, el cual es un ensayo muy práctico que da suficiente información de las características de agrietamiento de las mezclas asfálticas desde la formación de la grieta hasta su comportamiento de propagación, además es lo suficientemente sensible a los cambios en los contenidos de asfalto y tipo para discriminar entre distintos tipos de mezclas [Ref. 5].

\section{Descripción del equipo}

El equipo TTI Overlay Tester fue diseñado por Germann y Lytton, et al. [Ref. 3] a finales de los años 70, para simular el movimiento de juntas o grietas de apertura y cierre que es lo que causa que las grietas se reflejen en la superficie. Inicialmente existieron dos tipos de equipo cuya diferencia fue el tamaño del espécimen que se podía ensayar, en uno se ensayan especímenes pequeños de $375 \mathrm{~mm}$ (15 in) de largo por $75 \mathrm{~mm}$ (3 in) de ancho y altura variable mientras que el otro equipo los especímenes eran más grandes de $500 \mathrm{~mm}$ (20 in) de largo por $150 \mathrm{~mm}$ (6 in) de ancho y altura variable; el objetivo de estos equipos era probar adicionalmente el uso de geosintéticos como retardadores del reflejo de fisuras.

Posteriormente, Fujie Zhou y Tom Scullion adaptaron el equipo para ensayar especímenes más pequeños pues la fabricación de este tipo de viguetas era complicado, por lo que luego de la aparición del Compactador Giratorio se optó por acomodar especímenes de $150 \mathrm{~mm}$ de diámetro así como poder extraer núcleos de campo con esta dimensión. Se utilizó un programa de elemento finito para definir la dimensión mínima requerida para que los esfuerzos a tensión tuvieran toda la posible dimensión para desarrollarse y evitar los efectos de borde, las dimensiones establecidas son: $150 \mathrm{~mm}$ (6 in) de largo por $75 \mathrm{~mm}$ (3 in) de ancho y $38 \mathrm{~mm}$ (1,5 in) de altura.

El equipo consiste en un sistema electro-hidráulico que aplica cargas de tensión repetidas. Las partes más importantes se presentan en la figura 2, las cuales consisten en dos placas de acero base, una fija y la otra con movimiento horizontal o vertical para simular el movimiento de la grieta, dos placas ranuradas sobre las que se coloca el espécimen con epóxico, las placas se colocan separados con una distancia de $2 \mathrm{~mm}(0,08 \mathrm{in})$ entre ellas.

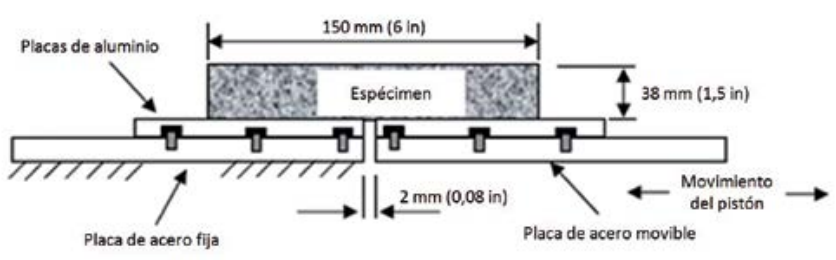

Figura 2. Esquema del equipo TTI Overlay Tester. Fuente: Zhou y Scullion, 2003. [Ref. 4].

El equipo además tiene que tener un dispositivo para medir el desplazamiento como un deformímetro pues el ensayo se realiza con control de deformación constante que puede variarse desde $0 \mathrm{~mm}$ hasta $2 \mathrm{~mm}$ pero se ha establecido $0,635 \mathrm{~mm}(0,025 \mathrm{in})$ como la deformación a aplicar pues esta es la cantidad que un pavimento de concreto hidráulico se desplaza cuando hay gradientes de temperatura de 14 ${ }^{\circ} \mathrm{C}$ en losas con juntas cada $4,5 \mathrm{~m}$.

También tiene una celda de carga para medir la carga de forma triangular que se puede aplicar de dos maneras:

- Método de una fase de carga (figura 3a): carga cíclica triangular sin reposo.

- Método de dos fases de carga (figura 3b): carga de relajación inicial para una deformación constante de 0,18 $\mathrm{mm}(0,007 \mathrm{in})$ para los 35 segundos iniciales del ensayo para luego aplicar la carga cíclica triangular hasta la falla. 
Desplazamiento

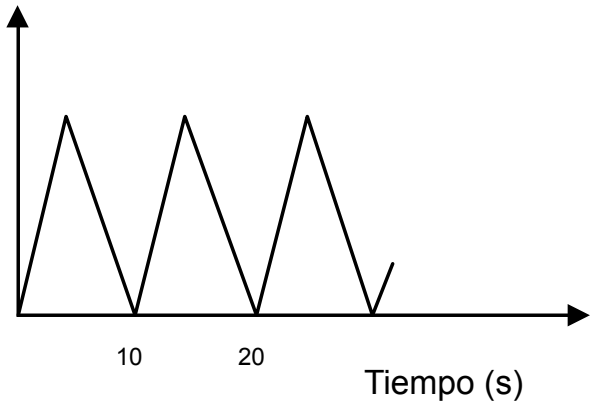

a. Una fase
Desplazamiento

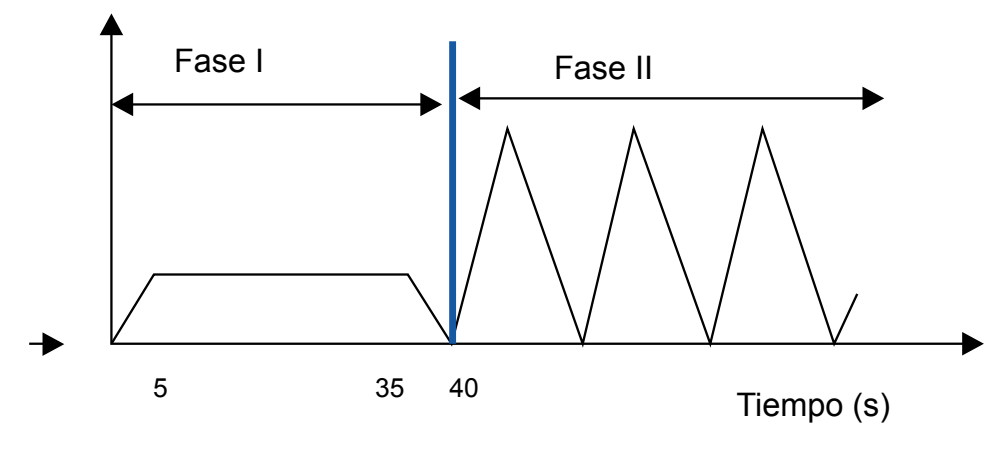

b. Dos fases

Figura 3. Esquema de los tipos de carga para el ensayo de overlay Fuente: Zhou y Scullion, 2005. [Ref. 3]

En el mercado mundial hay varias empresas que venden el equipo como Shedworks Inc. (http://www.shedworks.com/ pavementlabproducts/overlaytester.html) en el estado de Texas, Estados Unidos y en Cooper Technology en Inglaterra (http://www.cooper.co.uk/info/index.asp?page=texas_ overlay_tester_337). En Iraq construyeron un prototipo y se llama OTE para las viguetas largas. La empresa IPC Global en Australia (http://www.ipcglobal.com.au/product-range/ overlay-tester-kits.html) ofrece los accesorios para realizar el ensayo en el equipo AMPT.

El LanammeUCR en el año 2012 adquirió el equipo de ensayo AMPT (Asphalt Mixture Performance Tester), en el cual se pueden realizar varios ensayos de desempeño como módulo dinámico, ensayo de creep con carga dinámica, fatiga a tensión directa e indirecta. Este equipo trae los aditamentos para realizar el ensayo de Overlay, en este equipo el montaje del espécimen es vertical y la base fija es la superior tal como se muestra en la figura 4.

El laboratorio se dio a la tarea de implementar este nuevo ensayo y en este documento se detallan todos los pormenores, cuidados y problemas que se presentaron al realizar este proceso. La implementación de un nuevo método de ensayo requiere de un estudio bibliográfico exhaustivo, el cual aporta desde el nuevo conocimiento hasta evitar hacer labores que ya se probaron que no funcionan con el consecuente ahorro de tiempo y recursos.
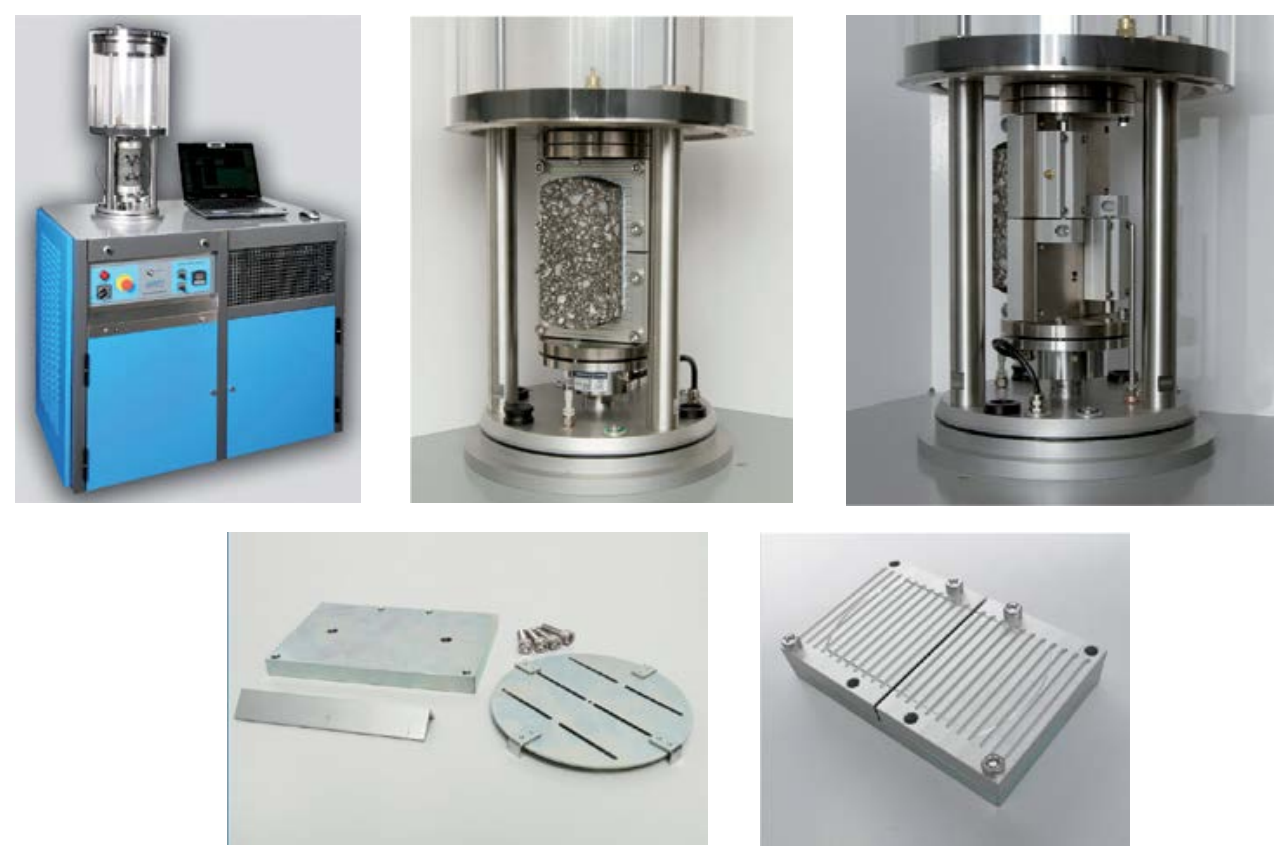

Figura 4. Equipo AMPT con los aditamentos para Overlay Fuente: AMPT OLTO512 IPC Global, 2012 [Ref. 7] 


\subsection{Datos y su interpretación}

El resultado más importante que se obtiene del ensayo de Overlay es la potencial resistencia al agrietamiento de la mezcla asfáltica, la cual se cuantifica en términos del número de ciclos que dura el espécimen en fallar, la falla se ha definido como una reducción del $93 \%$ de la carga medida en el primer ciclo bajo las condiciones del ensayo preestablecidas (figura 5).

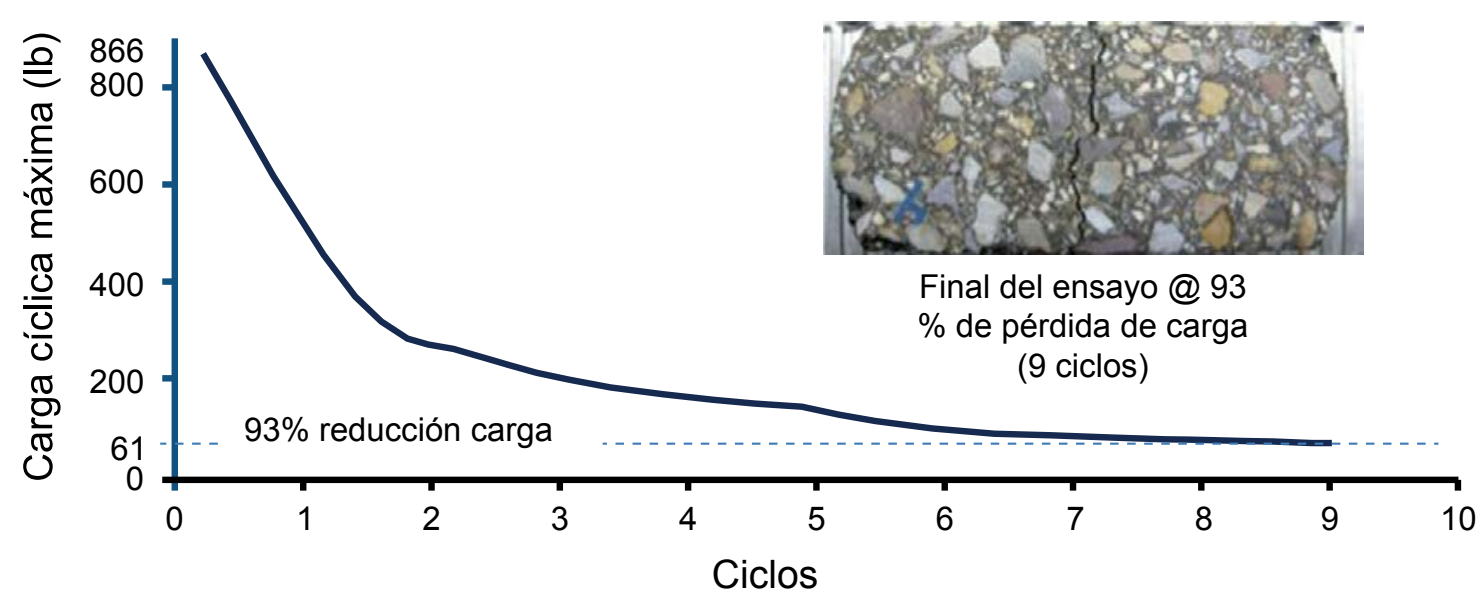

Figura 5. Falla del ensayo de Overlay

Fuente: Walubita et al., 2012 [Ref. 5]

Sin embargo, estos resultados tienen una variabilidad muy alta, por lo que algunos autores han expresado que hay más información que se puede obtener de los resultados del ensayo como el análisis e interpretación de los datos para obtener las características de la mecánica de la fractura.

Zhou y Scullion [Ref. 4] proponen que se analicen los datos para estudiar la duración de propagación de la grieta, graficando la carga y desplazamiento en función del tiempo. Indican que se puede establecer tres fases de comportamiento. En la figura 6 se presenta una salida del ensayo en forma gráfica, en la cual se pueden distinguir estas 3 etapas:

\section{Fase I: Inicio de la grieta y propagación inicial}

- En esta fase la carga y el desplazamiento tienen la misma forma, si el desplazamiento aumenta la carga también lo hace. Luego, la carga disminuye rápidamente cuando la fisura empieza a propagarse en el espécimen, en la figura 6 esta fase duró solo dos ciclos.
Fase II: Propagación tardía de la grieta

- En esta fase la carga máxima disminuye muy lentamente, no obstante, la carga máxima se mantiene en fase con el desplazamiento máximo.

Fase III: Falla del espécimen

- En esta fase la grieta se ha propagado completamente a través del espécimen. La carga máxima ocurre un poco antes del desplazamiento máximo. La magnitud de carga pequeña se asocia con la poca adhesión cuando el espacio entre placas está cerrado y las dos mitades del espécimen están unidas. Aún así, esta unión se puede deshacer fácilmente.

El espécimen mostrado en la figura 6 falló muy rápido. De acuerdo con las descripciones anteriores la duración de propagación de la grieta fue 6 ciclos. De acuerdo con este análisis, mientras se dé más duración en la propagación de la grieta mejor será el comportamiento de reflexión de grietas en sitio. 


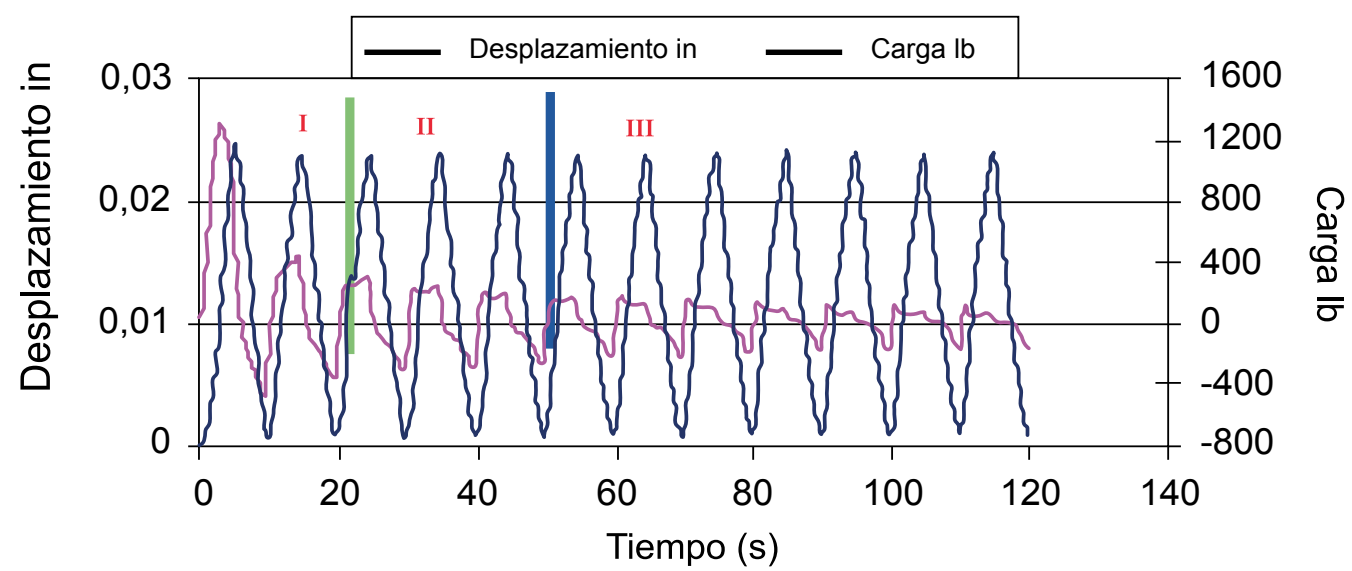

Figura 6. Datos graficados del ensayo de Overlay Fuente: Zhou y Scullion, 2003 [Ref. 4]

Adicionalmente, desde que Majidzadeh et al. (1970) introdujeron el concepto de la mecánica de la fractura en el campo de los pavimentos, este método se ha utilizado ampliamente para predecir el agrietamiento (fatiga, baja temperatura y reflejo) en pavimentos y su análisis. En el año 1963, Paris y Erdogan propusieron la ley de propagación de la grieta (ecuación 1), que describe la tasa de propagación de la fisura en mezclas asfálticas, aunque está basada en la mecánica de fractura lineal elástica.

$$
\frac{d c}{d N}=A(\Delta K)^{n}
$$

(ecuación 1)

donde:

$\mathrm{C}=\quad$ longitud de la grieta

$\mathrm{N}=\quad$ número de ciclos de carga

$\mathrm{dc} / \mathrm{dN}=$ tasa de propagación

$\mathrm{A}, \mathrm{n}=$ parámetros de fractura de la mezcla asfáltica determinados por medio de ensayos

$\Delta \mathrm{K}=$ cambio en el factor de intensidad de los esfuerzos (SIF) durante la carga y descarga

Algunos otros autores han desarrollado una serie de metodologías teóricas para determinar los parámetros A y $\mathrm{n}$, adicionalmente para determinar SIF se ha resuelto con la teoría del integral $\mathrm{J}$ viscoelástico. Con la ecuación 1 , se puede despejar el número de ciclos que se necesitan para que la grieta se propague en el espesor de una sobrecapa realizando una integral, pero se tienen que conocer los parámetros $\mathrm{A}, \mathrm{n}$ y SIF los cuales se ha probado que se pueden obtener directamente del ensayo de Overlay.

\section{Método de ensayo}

A continuación, se presenta un resumen del procedimiento de ensayo basado en el método estándar Tex-248-F [Ref. 1]
Procedimiento de ensayo de Overlay del Departamento de Transporte de Texas.

\subsection{Alcance}

El método determina la susceptibilidad de las mezclas asfálticas a fatigarse o el agrietamiento por reflexión con el cual se mide el número de ciclos a la falla. Las muestras se someten a tensión directa con carga repetida para especímenes fabricados en el laboratorio o núcleos obtenidos en el sitio.

\subsection{Equipo y materiales}

Para realizar este método de ensayo se requiere como equipo y materiales:

- Equipo Overlay Tester o accesorios del equipo AMPT: cámara ambientadora, deformímetro para medir el desplazamiento de la placa, celda de carga, placas base ranuradas y plantilla para el montaje del espécimen (figura 4)

- Compactador Giratorio Superpave ${ }^{\circledR}$

- Moldes de $152,4 \mathrm{~mm}$ de diámetro o extractora de núcleos con broca de 152,4 mm de diámetro

- Epóxico de dos fases con una resistencia a tensión y cortante después de 24 horas de 4,1 MPa (600 psi) y 13,8 MPa (2 000 psi) respectivamente.

- Balanza, cinta adhesiva con un espesor de $6,25 \mathrm{~mm} \mathrm{(1/4}$ in) y pintura. 


\subsection{Método de ensayo resumido}

\subsubsection{Especímenes de ensayo:}

Se pueden ensayar especímenes fabricados en el laboratorio o núcleos extraídos de sitio. Para los especímenes de laboratorio, tienen que tener un diámetro de $152,4 \mathrm{~mm}$ y una altura de $(127 \pm 5) \mathrm{mm}$ para poder cortar 2 briquetas desde el centro cuyas dimensiones se detallan más adelante o también de $(62,5 \pm 5,0) \mathrm{mm}$ para cortar 1 briqueta. El porcentaje de vacíos de las briquetas debe estar entre $(7 \pm 1) \%$, pero para obtener este valor los especímenes compactados deben tener un porcentaje de vacíos mayor de aproximadamente $1,5 \%$.

Cuando se vayan a ensayar núcleos de sitio estos deben tener una altura mínima de $38 \mathrm{~mm}$ luego de cortados, no tienen que cumplir con una densidad específica.

Se requiere un mínimo de 3 briquetas pero lo ideal es que sean 6 .

\subsubsection{Cortado de briquetas:}

Se tienen que cortar los especímenes cilíndricos para obtener una briqueta con las dimensiones de la figura 7 . Se puede utilizar un plantilla para marcar el espécimen.

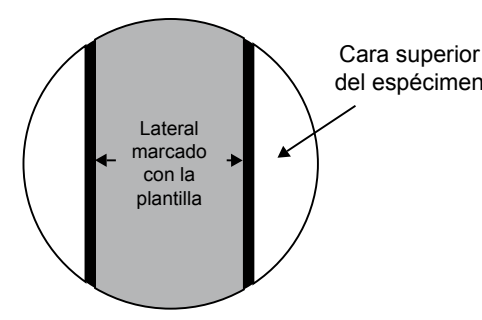

Marcado de la cara del espécimen con plantilla

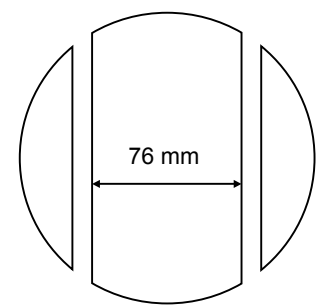

Cortado de los lados del espécimen

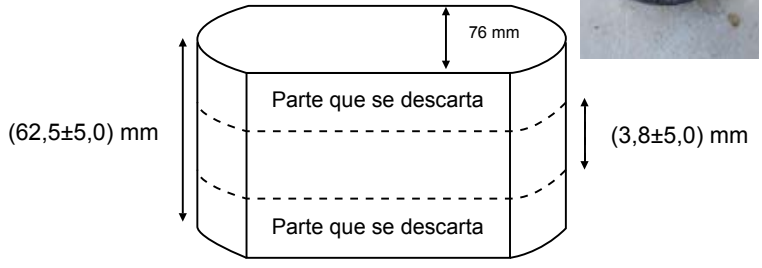

Figura 7. Cortado de 1 briqueta para ensayo de Overlay Fuente: Tex 248-F, 2009 [Ref. 1]
Se corta los lados laterales siguiendo las líneas marcadas, esto se descarta. Luego se cortan la parte superior e inferior del espécimen para sacar desde el centro una briqueta de $(38,0 \pm 0,5) \mathrm{mm}$.

Si se van a cortar 2 especímenes de un mismo cilindro estos se deben cortar de tal manera que las caras cortadas del centro no sean coincidentes sino que tengan una distancia de separación como se muestra en la figura 8 , las partes $\sin$ relleno se descartan.

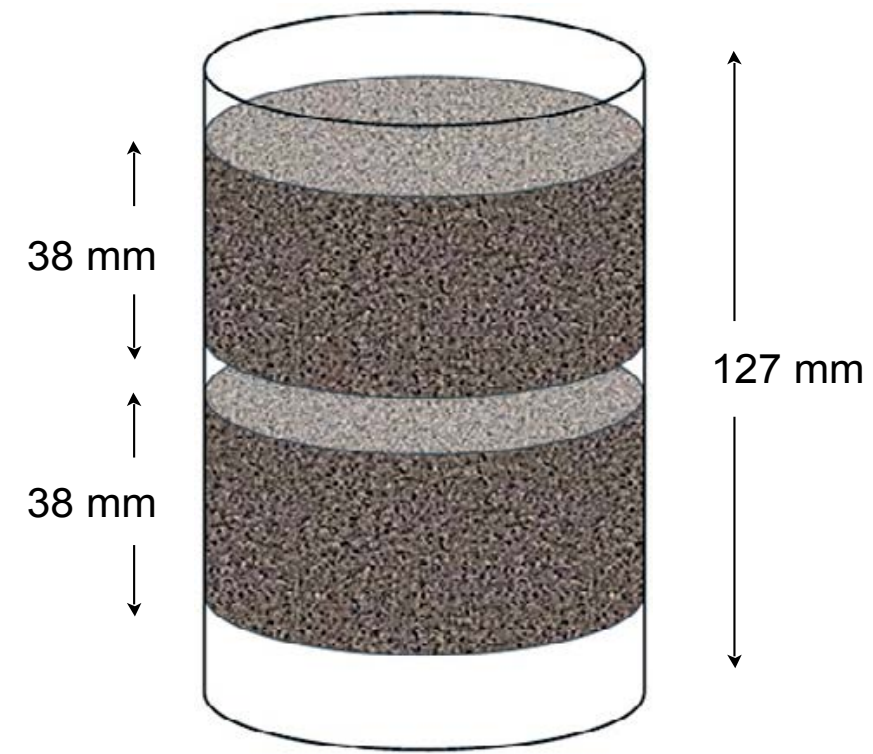

Figura 8. Cortado de 2 briquetas para ensayo de Overlay Fuente: Walubita et al, 2012 [Ref. 5]

\subsubsection{Densidad de las briquetas:}

Después de cortadas las briquetas se tiene que medir su densidad la cual debe cumplir con el contenido de vacíos de $(7 \pm 1) \%$, esta medida se hace de la siguiente manera: se toma el peso sumergido y peso superficie saturada seca luego se ponen a secar en un horno a $(40 \pm 3){ }^{\circ} \mathrm{C}$ durante 12 horas hasta conseguir peso constante el cual se define como, el peso que no cambia en más de $0,05 \%$ en intervalos de 2 horas de secado. Los especímenes que no cumplan con el contenido de vacíos se tienen que descartar. Se tiene que medir las dimensiones de la briqueta, ancho, alto y largo.

\subsubsection{Montaje de la briqueta en los platos base:}

Los dos platos base se montan sobre la plantilla de montaje, estos platos se deben ajustar con los tornillos de tal manera que la separación entre ambos platos sea de $2 \mathrm{~mm}$, esta separación se debe medir con una galga para 
asegurar que la separación es la misma a lo largo de toda la distancia (figura 9a). Una vez que se tiene esta separación, se debe pegar un pedazo de cinta adhesiva a lo largo de la separación, la cual debe tener un largo de 102 mm (Figura 9c). Esto se tiene que realizar con mucho cuidado para que la cinta quede centrada y sin burbujas.

Para una mejor observación de las grietas que se producirán es recomendable pintar de blanco el área central del espécimen (figura 9b).

Se tiene que preparar el epóxico de acuerdo con las indicaciones del fabricante. Se tiene que cubrir la mayor parte de los platos base con el epóxico, utilizar aproximadamente 16 gramos de epóxico que es suficiente cantidad para que no haya un exceso del mismo y se extienda fuera cuando se aplique la carga, también hay que poner epóxico sobre la cara de la briqueta evitando poner material sobre el centro.
Luego de varias pruebas observamos que es mejor marcar el centro del espécimen y colocar la cinta adhesiva para evitar que el epóxico quede en esta área (Figura 9c), adicionalmente es mejor medir 8 gramos de un lado y los otros 8 gramos en el otro lado para mezclar el epóxico y así evitar el desperdicio y derrame (figura 9d), luego se pasa la mayor parte a los platos base (figura 9e).

Pegar la briqueta a los platos base asegurándose que quede alineada, utilizar la regla para alinearlo (figura 9f).

Finalmente, se coloca una pesa de $4,5 \mathrm{~kg}$ sobre la briqueta para asegurar un contacto total a los platos base. Se tiene que dejar secar el epóxico el tiempo indicado por el fabricante, una vez que el epóxico esté seco quitar la pesa, esto se puede dejar toda la noche (figura 9g).

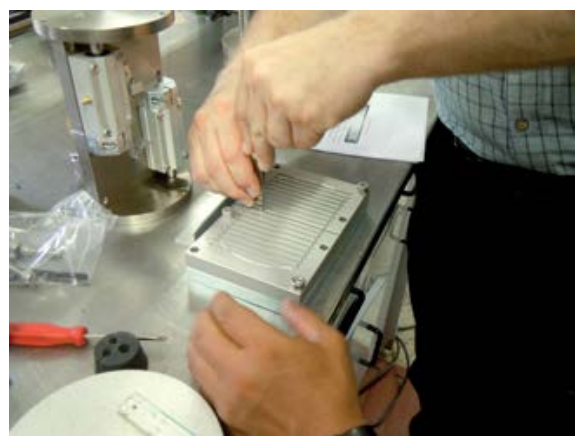

(a)

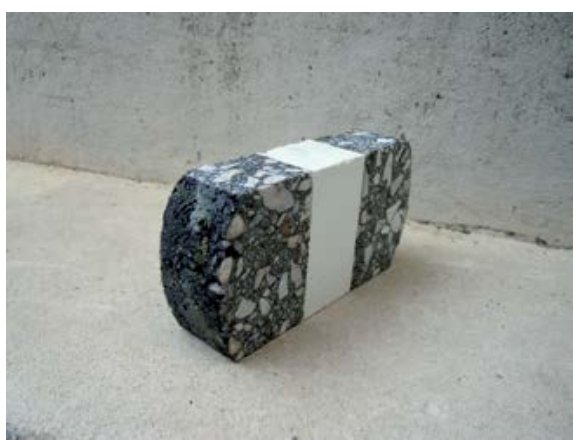

(b)

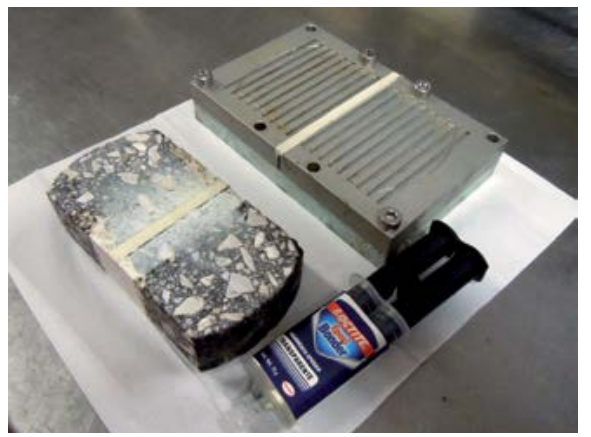

(c)

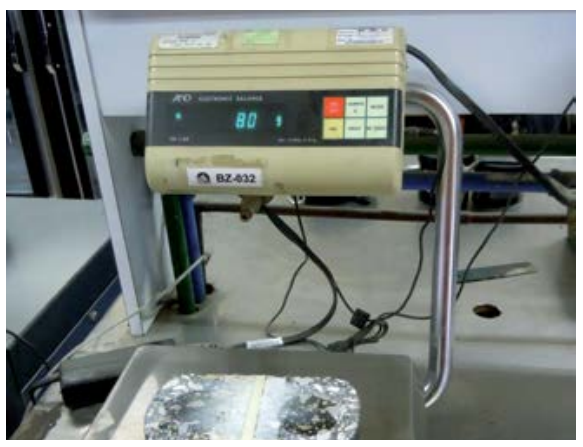

(d)

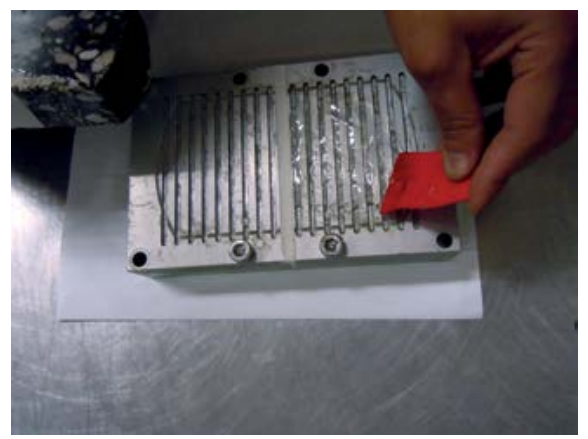

(e)

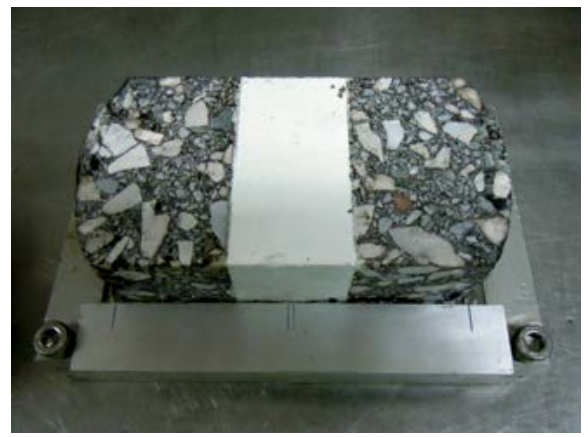

(f)

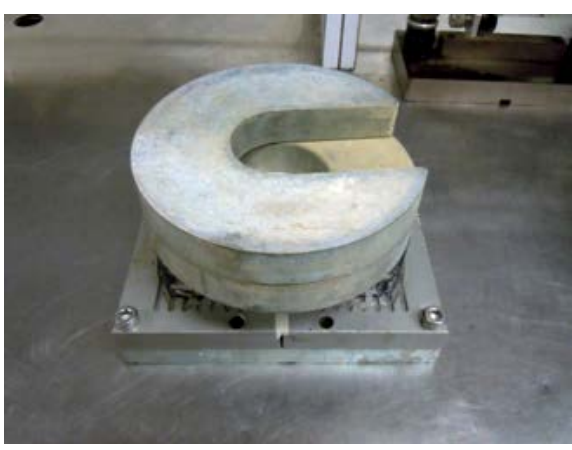

(g)

a. Separación entre platos base

b. Pintura en la parte central de la briqueta

c. Cinta en el centro del espécimen y en los platos base d. Colocación epóxico

e. Colocación epóxico en los platos base

f. Alineamiento de la briqueta

g. Pesa de $4.5 \mathrm{~kg}$

Figura 9. Montaje de briqueta 


\subsection{5}

Temperatura de ensayo:

Una vez que la briqueta está bien pegada a los platos base, se coloca sobre las muelas del equipo y todo el dispositivo se coloca dentro de la cámara ambientadora del equipo, la cual debe programarse para una temperatura de ensayo de $(25,0 \pm 0,5)^{\circ} \mathrm{C}$. En el montaje de la muela de ensayo se tiene que aplicar un torque de $20 \mathrm{~N} \cdot \mathrm{m}$ (15 lb·in) en cada tornillo para asegurar la uniformidad entre especímenes (figura 10). Se tiene que colocar el deformímetro para que su recorrido sea el adecuado para medir la distancia de abertura durante el ensayo (figura 11). Se debe dejar ambientar el espécimen por al menos 1 hora antes de iniciar el ensayo.
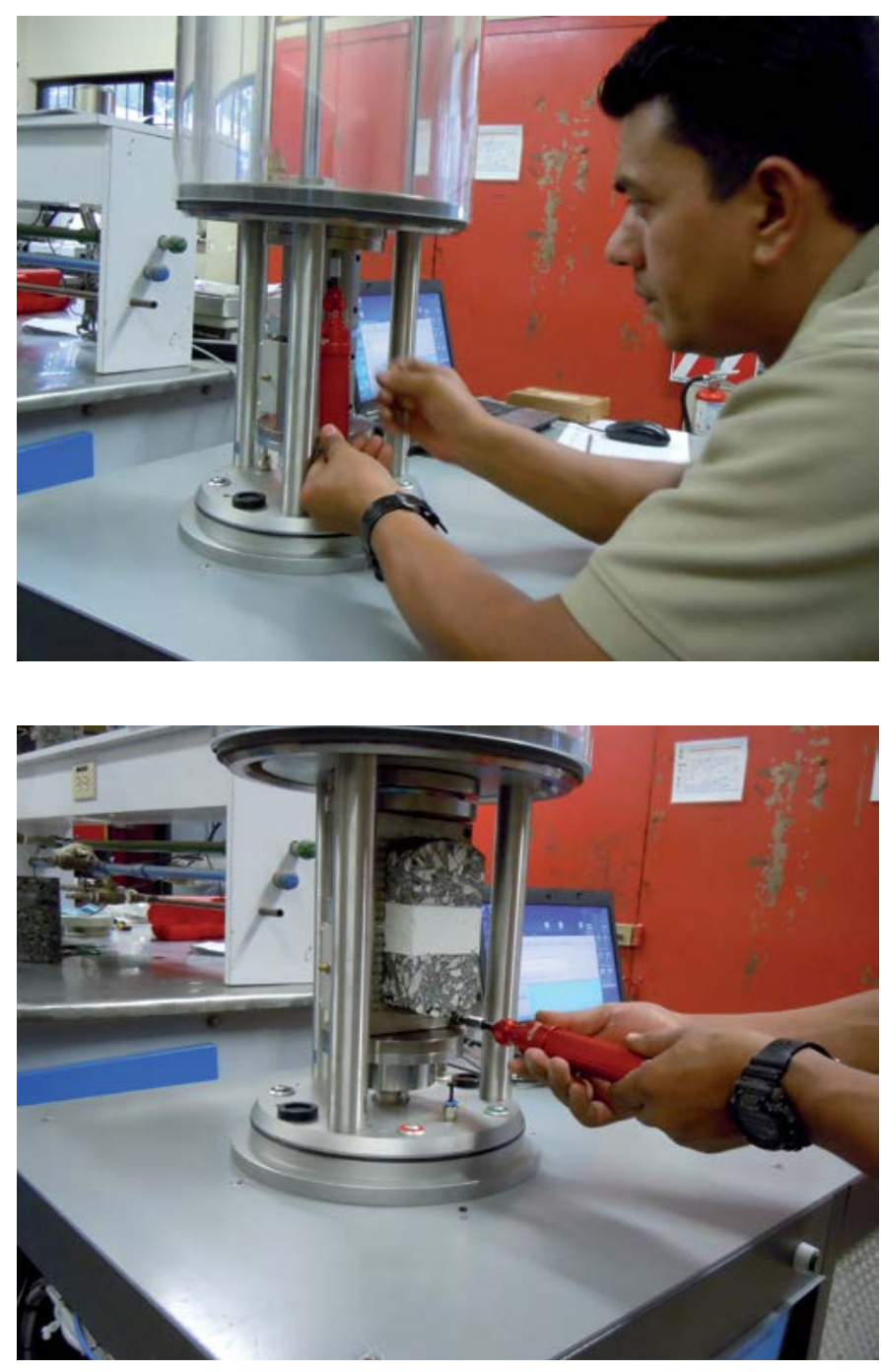

Figura 10. Montaje de la muela del ensayo en el equipo

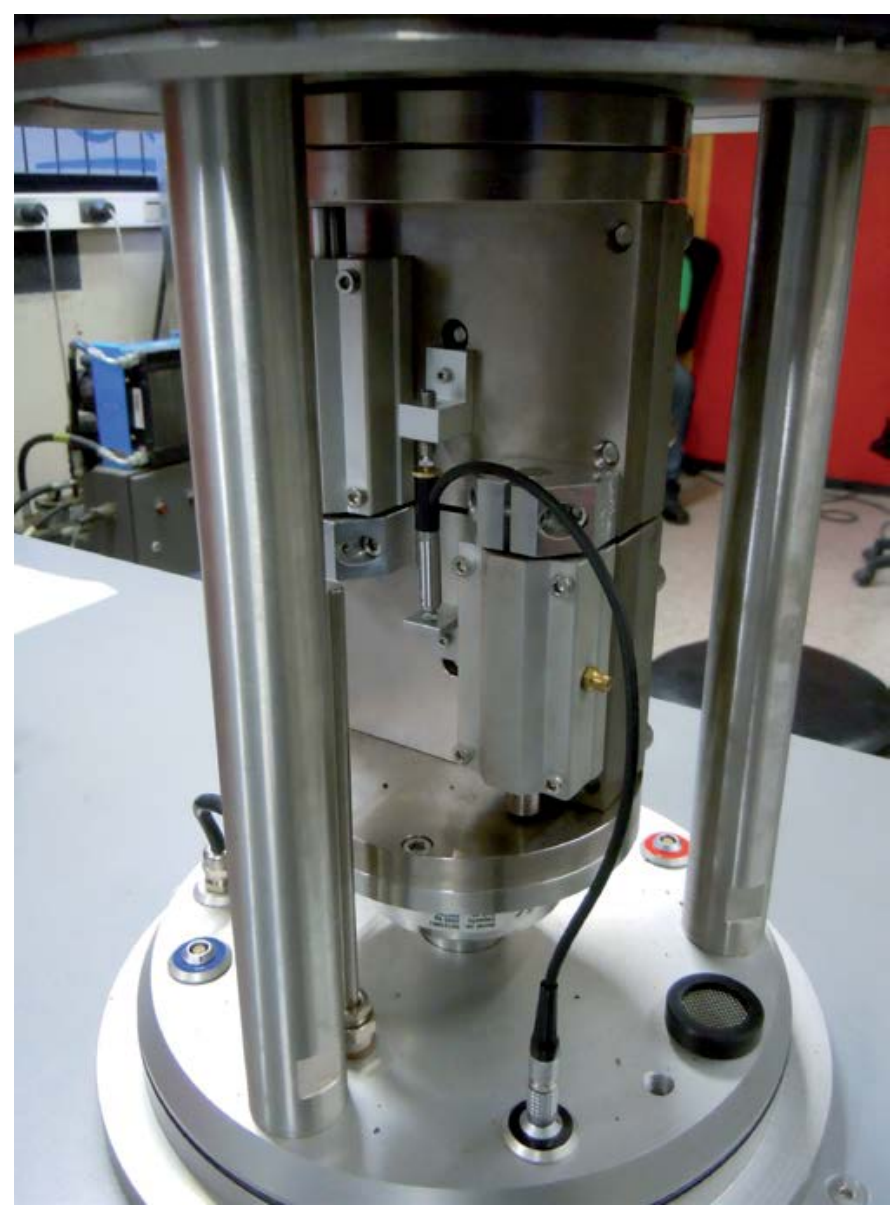

Figura 11. Montaje del deformímetro

\subsubsection{Software:}

El software para realizar el ensayo en el equipo AMPT es el UTS 036 (figura 12). En este se tiene que introducir toda la información de la muestra como el proyecto, operador, identificación de la muestra y dimensiones de la briqueta.

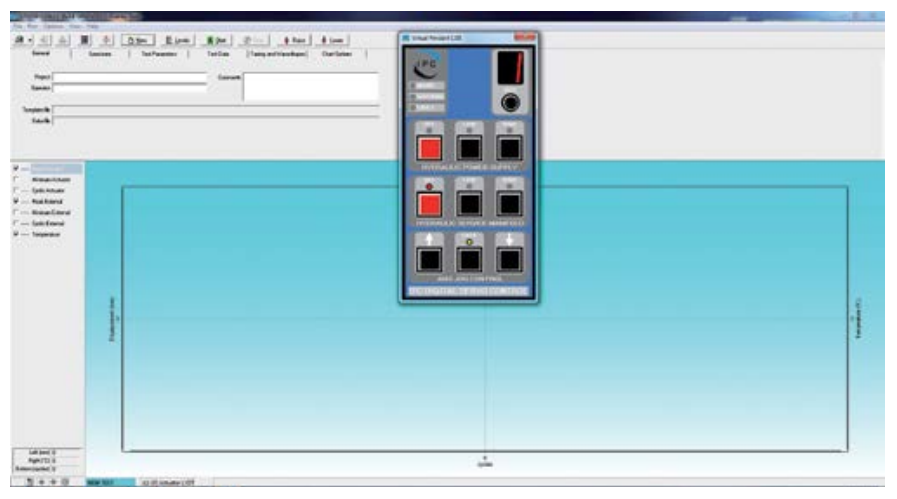

Figura 12. Software para realizar el ensayo de Overlay 
Se tiene que iniciar el ensayo y se tiene que alimentar los parámetros del ensayo los cuales incluyen: forma de carga: triangular, la temperatura de ensayo: $25^{\circ} \mathrm{C}$, el periodo de relajación antes del ensayo: 10 min, la abertura de separación: $0,635 \mathrm{~mm}$, tiempo del pulso de carga: 10 segundos, frecuencia de carga: $0,1 \mathrm{~Hz}$, finalmente los criterios de finalización del ensayo: el porcentaje de reducción de la carga inicial: $93 \%$ y número máximo de ciclos de carga: 1200 . El ensayo se debe realizar con control de esfuerzo o carga (figura 13).

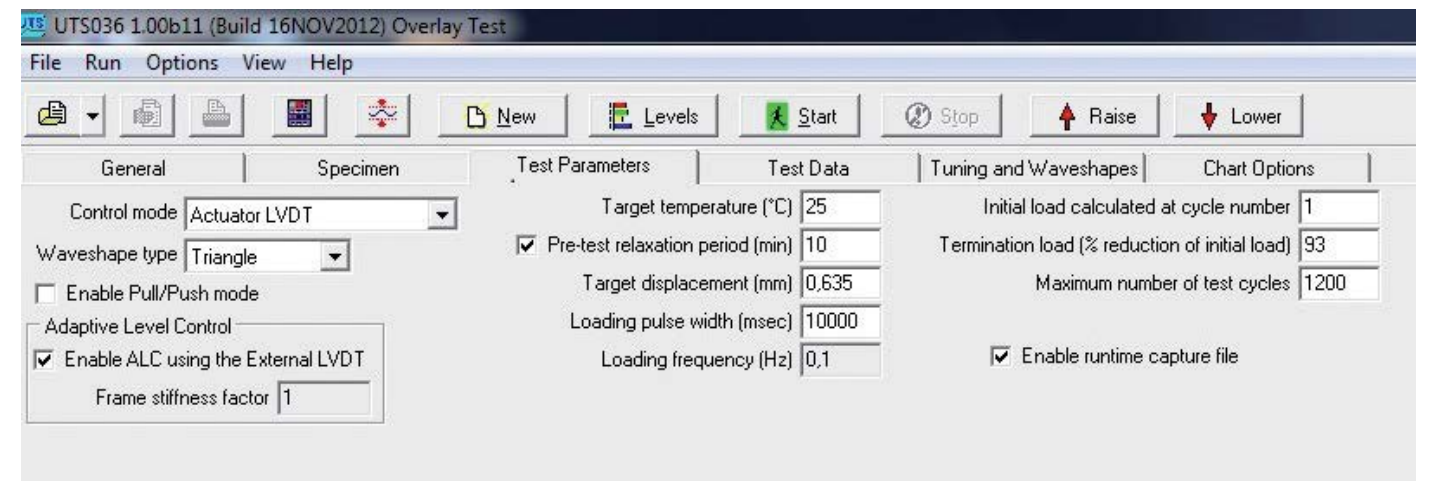

Figura 13. Parámetros para realizar el ensayo de Overlay

El ensayo termina cuando se dé uno de los dos criterios anteriores. Se procede a desmontar el espécimen.

\subsection{Resultados obtenidos del ensayo}

Se tiene que reportar la siguiente información:

- Identificación de la muestra.

- Descripción de la muestra: mezcla preparada en el laboratorio, tipo de mezcla o espécimen obtenido de sitio, localización del punto de muestreo.

- Densidad de las briquetas ensayadas.

- Parámetros de ensayo, listados en el punto 3.3.7 de este artículo.

- Carga inicial.

- Carga final.

- Porcentaje de pérdida de carga.

- Número de ciclos a la falla.

- Promedio, desviación estándar y coeficiente de variación.

- Número de grietas observadas: cero, una o más (figura 14).

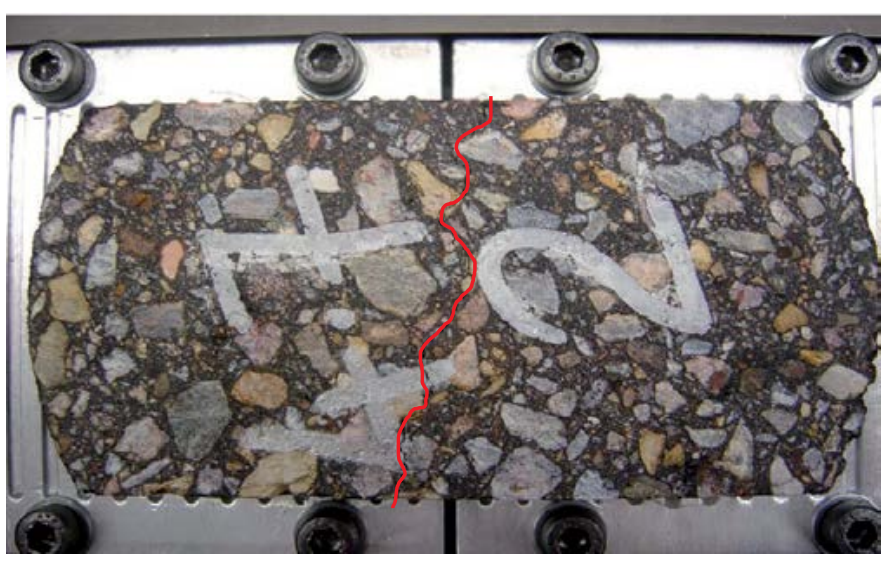

a. Una grieta

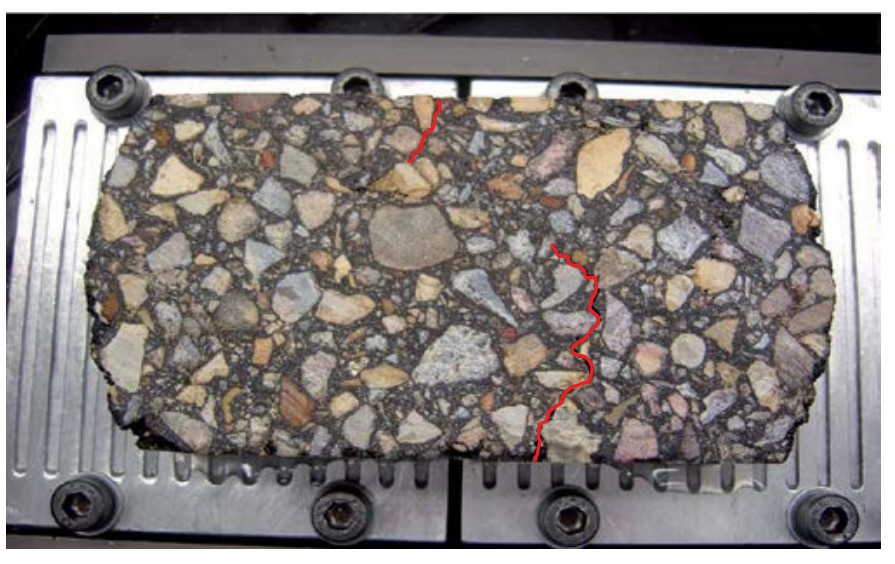

b. Dos grietas

Figura 14. Grietas observadas luego del ensayo Fuente: Walubita et al, 2012 [Ref. 1] 


\section{Test Data}

Start Date and Time: Tuesday, July 23, 2013, at 9:43:00 AM Loading time (hh:mm:ss): 00:43:40
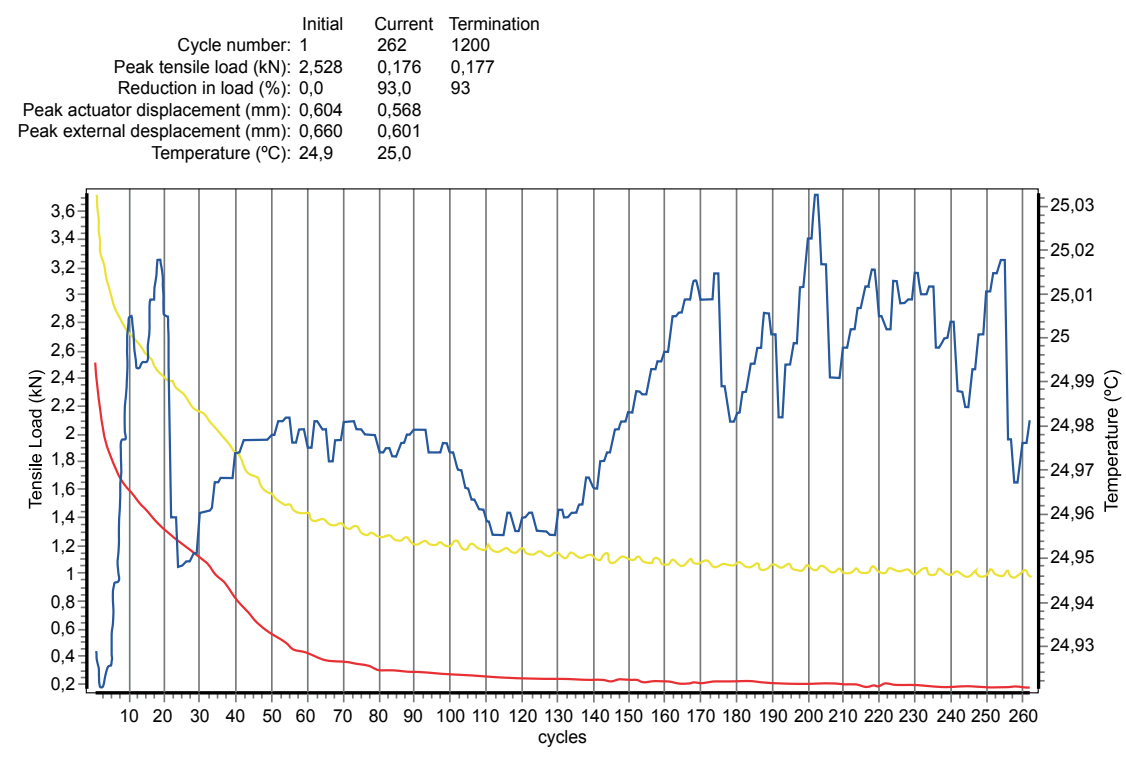

- Peak Load - Cyclic Load - Temperature

Figura 15. Salida típica del ensayo de Overlay

A continuación se presentan los resultados obtenidos para dos muestras de mezcla asfáltica tomadas en planta, una sin modificar y otra modificada con látex. Estas muestras se utilizaron con el objetivo de implementar el método de ensayo en el LanammeUCR.

\subsubsection{Mezcla sin modificar (SM):}

Los especímenes se compactaron a un nivel de vacíos de aire de 9,0\% para esperar un 7,0 \% en las briquetas pero se obtuvo una alta variación en el porcentaje de vacíos de aire, aún así se continuó con el montaje del ensayo y en la tabla 1 se presentan todos los resultados obtenidos.

Tabla 1: Resultados de la muestra de mezcla asfáltica sin modificar (SM).

\begin{tabular}{|c|c|c|c|c|c|c|c|c|c|}
\hline $\begin{array}{c}N^{\circ} \text { espécimen } \\
(\mathrm{Hz})\end{array}$ & $\begin{array}{l}\text { Porcentaje } \\
\text { de vacíos }\end{array}$ & Temp. $\left({ }^{\circ} \mathrm{C}\right)$ & $\begin{array}{l}\text { Frecuencia } \\
\quad(\mathrm{Hz})\end{array}$ & $\begin{array}{c}\text { Desplazamiento } \\
\text { objetivo }(\mathrm{mm})\end{array}$ & $\begin{array}{l}\text { Carga inicial } \\
\text { (kN) }\end{array}$ & $\begin{array}{l}\text { Carga final } \\
\text { (kN) }\end{array}$ & $\begin{array}{l}\text { Porcentaje de } \\
\text { pérdida en la } \\
\text { carga (\%) }\end{array}$ & $\begin{array}{l}\text { Número de } \\
\text { ciclos a la } \\
\text { falla (ciclos) }\end{array}$ & $\begin{array}{l}\text { Cantidad } \\
\text { de grietas } \\
\text { (unidades) }\end{array}$ \\
\hline $24 \mathrm{~A}$ & 8,7 & 25,0 & 0,1 & 0,6 & 2,258 & 0,176 & 93,0 & 262 & 1 \\
\hline $24 \mathrm{~B}$ & 6,8 & 25,0 & 0,1 & 0,6 & 2,699 & 0,188 & 93,0 & 233 & 1 \\
\hline $25 \mathrm{~A}$ & 8,3 & 25,0 & 0,1 & 0,6 & 2,899 & 0,202 & 93,0 & 218 & 1 \\
\hline $25 \mathrm{~B}$ & 7,4 & 25,0 & 0,1 & 0,6 & 2,610 & 0,178 & 93,0 & 69 & 0 \\
\hline $26 \mathrm{~A}$ & 6,7 & 25,0 & 0,1 & 0,6 & 3,035 & 0,208 & 93,1 & 134 & 1 \\
\hline $26 \mathrm{~B}$ & 7,4 & 25,0 & 0,1 & 0,6 & 2,699 & 0,184 & 93,2 & 84 & 0 \\
\hline Promedio & 7,5 & 25,0 & 0,1 & 0,6 & 2,745 & 0,189 & 93,1 & 167 & -- \\
\hline Desvest & 0,8 & -- & -- & -- & 0,188 & 0,013 & 0,1 & 82 & -- \\
\hline cv & 10,9 & -- & -- & -- & 6,86 & 6,87 & 0,1 & 49 & -- \\
\hline
\end{tabular}




\subsubsection{Mezcla modificada con látex (M):}

Los especímenes se compactaron a un nivel de vacíos de aire de 9,4\% para esperar un 7,0 \% en las briquetas pero se obtuvo una mejor variación en el porcentaje de vacíos de aire y en la tabla 2 se presentan todos los resultados obtenidos.

Tabla 2: Resultados de la muestra de mezcla asfáltica modificada con latex (M).

\begin{tabular}{|c|c|c|c|c|c|c|c|c|c|}
\hline $\begin{array}{c}N^{\circ} \text { espécimen } \\
(\mathrm{Hz})\end{array}$ & $\begin{array}{l}\text { Porcentaje } \\
\text { de vacíos }\end{array}$ & Temp. $\left({ }^{\circ} \mathrm{C}\right)$ & $\begin{array}{l}\text { Frecuencia } \\
(\mathrm{Hz})\end{array}$ & $\begin{array}{c}\text { Desplazamiento } \\
\text { objetivo }(\mathrm{mm})\end{array}$ & $\begin{array}{l}\text { Carga inicial } \\
\text { (kN) }\end{array}$ & $\begin{array}{l}\text { Carga final } \\
\text { (kN) }\end{array}$ & $\begin{array}{l}\text { Porcentaje de } \\
\text { pérdida en la } \\
\text { carga (\%) }\end{array}$ & $\begin{array}{c}\text { Número de } \\
\text { ciclos a la } \\
\text { falla (ciclos) }\end{array}$ & $\begin{array}{l}\text { Cantidad } \\
\text { de grietas } \\
\text { (unidades) }\end{array}$ \\
\hline $24 \mathrm{~A}$ & 7,9 & 25,0 & 0,1 & 0,6 & 1,503 & 0,274 & 81,8 & 1200 & 1 \\
\hline $24 \mathrm{~B}$ & 7,6 & 25,2 & 0,1 & 0,6 & 1,538 & 0,249 & 83,8 & 1200 & 1 \\
\hline $25 \mathrm{~A}$ & 8,1 & 25,3 & 0,1 & 0,6 & 2,159 & 0,456 & 78,9 & 1200 & 1 \\
\hline $25 \mathrm{~B}$ & 7,6 & 25,0 & 0,1 & 0,6 & 2,178 & 0,438 & 79,9 & 1200 & 1 \\
\hline $26 \mathrm{~B}$ & 7,6 & 25,0 & 0,1 & 0,6 & 2,146 & 0,375 & 82,5 & 1200 & 1 \\
\hline Promedio & 7,8 & 25,1 & 0,1 & 0,6 & 1,965 & 0,374 & 81,2 & 1200 & 1 \\
\hline Desvest & 0,2 & 0,2 & -- & -- & 0,347 & 0,092 & 1,8 & 0 & -- \\
\hline CV & 2,8 & 0,6 & -- & -- & 17,66 & 24,63 & 2,3 & 0 & -- \\
\hline
\end{tabular}

De acuerdo con los resultados anteriores, se puede observar que el aporte del látex en la mezcla es significativo para la reducción de la susceptibilidad al agrietamiento.

\subsection{Criterios de precisión y exactitud}

Los criterios de precisión permiten determinar la validez de los resultados obtenidos. Para este método se ha establecido un coeficiente de variación menor o igual al 30 $\%$ para 3 briquetas de la misma mezcla, que es un valor con una alta robustez.

Preliminarmente, se ha establecido una desviación estándar de 65 ciclos para 3 briquetas basada en un estudio que incluyó 150 réplicas y 50 tipos de mezclas asfálticas que incluyeron mezcla densas finas y gruesas, mezclas gruesas, mezclas discontinuas y mezclas drenantes.

En cuanto al criterio de exactitud no se ha definido hasta el momento un material patrón que sirva como valor de referencia para establecer la exactitud del método.

\section{Factores que afectan la repetibilidad y sensibilidad del ensayo}

Debido a la alta variabilidad del método, se han reconocido varios factores que afectan la uniformidad de los resultados. Es de suma importancia reconocer esto sobre todo cuando se quiere implementar el método a nivel de laboratorio. Esto ha resultado en que varios autores se han dado a la tarea de realizar análisis de sensibilidad para establecer con claridad cuáles factores son los más relevantes, en este artículo se presenta un estudio realizado en la Universidad de Texas A\&M y el Instituto de Transporte de Texas [Ref. 5] en el cual establecieron 9 variables como las más significativas y estudiaron muestras de planta así como muestras preparadas en el laboratorio.

\subsection{Compactación de especímenes y número de briquetas a ensayar}

Para minimizar el desperdicio de material a la hora de cortar las briquetas y optimizar la carga de trabajo, los autores recomendaron que se compacte un espécimen de $127 \mathrm{~mm}$ de altura para cortar 2 briquetas o compactar un espécimen de $63,5 \mathrm{~mm}$ de altura para cortar 1 briqueta. Las briquetas se tienen que cortar de manera que salgan desde el centro de la altura.

El número apropiado de replicas a ensayar es crítico para asegurar la caracterización estadística correcta de la propiedad de resistencia al agrietamiento de la mezcla asfáltica. Se ensayaron 5 muestras secas al aire y otras 5 secas al horno con tres tipos de mezclas, en la tabla 3 se presentan los resultados obtenidos, de los cuales se concluye que se ensayen de 4 a 5 briquetas y de estas calcular el coeficiente de variación de las 3 briquetas que dé más bajo. 
Tabla 3: Efecto del número de réplicas ensayadas Fuente: Walubita et al. Tabla 5-1. 2012 [Ref. 5]

\begin{tabular}{|c|c|c|c|c|c|}
\hline \multirow{2}{*}{$\begin{array}{c}\text { Tipo de } \\
\text { mezcla }\end{array}$} & \multirow{2}{*}{$\begin{array}{c}\text { Método } \\
\text { de } \\
\text { secado }\end{array}$} & \multicolumn{4}{|c|}{$\begin{array}{c}\text { Coeficiente de variación } \\
\text { (promedio de los ciclos de falla), \% }\end{array}$} \\
\cline { 3 - 6 } & $\begin{array}{c}\text { Todos } \\
\text { los 5 }\end{array}$ & 4 mejores & 3 mejores & 2 mejores \\
\hline \multirow{2}{*}{$\begin{array}{c}\text { Tipo D } \\
(5.2 \% \text { CA })\end{array}$} & Aire & $68,9(119)^{\star}$ & $21,6(83)$ & $8,5(92)$ & $2,9(96)$ \\
\cline { 2 - 6 } & Horno & $34,5(122)$ & $25,1(135)$ & $6,3(118)$ & $4,3(115)$ \\
\hline \multirow{2}{*}{$\begin{array}{c}\text { Tip D } \\
(5.5 \% \text { CA) }\end{array}$} & Aire & $61,2(538)$ & $40,6(645)$ & $26,1(527)$ & $12,7(600)$ \\
\cline { 2 - 6 } & Horno & $46,7(396)$ & $36,5(450)$ & $19,5(520)$ & $0,7(579)$ \\
\hline \multirow{2}{*}{$\begin{array}{c}\text { Tipo D } \\
(4.9 \% \text { CA) }\end{array}$} & Aire & $57,2(187)$ & $43,3(217)$ & $31,6(176)$ & $19,1(204)$ \\
\cline { 2 - 6 } & Horno & $61,2(392)$ & $34,7479)$ & $7,5(560)$ & $0,1(536)$ \\
\hline \multirow{2}{*}{$\begin{array}{c}\text { Tipo CAM } \\
(6.7 \% \text { CA) }\end{array}$} & Aire & $17,2(928)$ & $0,0(1000)$ & $0,0(1000)$ & $0,0(1000)$ \\
\cline { 2 - 6 } & Horno & $18,4(856)$ & $14,9(903)$ & $6,0(967)$ & $0,0(1000)$ \\
\hline \multirow{2}{*}{$\begin{array}{c}\text { Tipo C } \\
(5.0 \% \text { CA) }\end{array}$} & Aire & $41,7(20)$ & $32,1(22)$ & $22,3(25)$ & $10,1(28)$ \\
\cline { 2 - 6 } & Horno & $50,4(36)$ & $31,2(29)$ & $15,6(24)$ & $2,7(27)$ \\
\hline
\end{tabular}

* Los valores entre paréntesis indican el promedio de ciclos.

De acuerdo con este criterio se tiene que escoger los tres resultados que tengan la menor variabilidad, por lo tanto, los resultados de la mezcla sin modificar las tres primeras briquetas son los que tienen esta característica con un CV de 9,4\% pero dos de las briquetas no cumplen con el contenido de vacíos, por lo tanto, se reporta en la tabla 4 la siguiente combinación. secado: secado al aire con ayuda de un ventilador durante toda la noche, secado al horno a $40{ }^{\circ} \mathrm{C}$ durante 12 horas y secado con el equipo de secado al vacío, luego de esta comparación se llegó a la conclusión de que el secado al horno es el método más adecuado pero este secado no debe exceder las 24 horas (figura 16).

\subsection{Periodo de reposo antes del ensayo}

El envejecimiento y fragilidad de la mezcla asfáltica es un proceso que toma mucho tiempo, el envejecimiento se puede dar por el proceso de volatilización y oxidación del asfalto. Debido a esto no se espera que a nivel de laboratorio la duración de la preparación de las briquetas sea un factor considerable, normalmente se toma entre 3 y 5 días todo el proceso desde la preparación de la mezcla hasta el secado de las briquetas. Aún así, se estudió cuánto puede afectar el periodo de reposo de la briqueta antes de ser ensayada, por lo que se almacenaron las muestras a temperatura ambiente por $3,5,7,9,11,13,30$ y 60 días. De acuerdo con los resultados obtenidos no se debe dejar reposar las briquetas más de 5 días.

Tabla 4. Resultados de la muestra de mezcla asfáltica sin modificar (SM) escogidos.

\begin{tabular}{|c|c|c|c|c|c|c|c|c|c|}
\hline $\begin{array}{c}N^{\circ} \text { espécimen } \\
(\mathrm{Hz})\end{array}$ & $\begin{array}{l}\text { Porcentaje } \\
\text { de vacíos }\end{array}$ & Temp. $\left({ }^{\circ} \mathrm{C}\right)$ & $\begin{array}{l}\text { Frecuencia } \\
\quad(\mathrm{Hz})\end{array}$ & $\begin{array}{c}\text { Desplazamiento } \\
\text { objetivo }(\mathrm{mm})\end{array}$ & $\begin{array}{l}\text { Carga inicial } \\
\text { (kN) }\end{array}$ & $\begin{array}{l}\text { Carga final } \\
\text { (kN) }\end{array}$ & $\begin{array}{c}\text { Porcentaje de } \\
\text { pérdida en la } \\
\text { carga }(\%)\end{array}$ & $\begin{array}{c}\text { Número de } \\
\text { ciclos a la } \\
\text { falla (ciclos) }\end{array}$ & $\begin{array}{r}\text { Cantidad } \\
\text { de grietas } \\
\text { (unidades) }\end{array}$ \\
\hline 11B SM & 6,8 & 25,0 & 0,1 & 0,6 & 2,699 & 0,188 & 93,0 & 233 & 1 \\
\hline $12 \mathrm{~A} \mathrm{SM}$ & 8,3 & 25,0 & 0,1 & 0,6 & 2,899 & 0,202 & 93,0 & 218 & 1 \\
\hline $13 \mathrm{~A} \mathrm{sm}$ & 6,7 & 25,0 & 0,1 & 0,6 & 3,035 & 0,208 & 93,1 & 134 & 1 \\
\hline Promedio & 7,2 & 25,0 & 0,1 & 0,6 & 2,878 & 0,199 & 93,0 & 195 & 1 \\
\hline cv & 12,4 & -- & -- & -- & 5,87 & 5,15 & 0,1 & 27 & -- \\
\hline
\end{tabular}

\subsection{Método de secado de las briquetas}

El método de Texas indica que las briquetas se sequen a una temperatura de $(60 \pm 3)^{\circ} \mathrm{C}$, pero esta temperatura de secado está muy cercana a la temperatura alta del Grado de Desempeño para un asfalto de tipo PG 64 y es mayor para un asfalto tipo PG 58, lo que puede ocasionar un sobrecalentamiento y oxidar de manera excesiva la mezcla. Es por esta razón, que es importante establecer un método de secado que no afecte de manera negativa a la mezcla asfáltica, por lo que se estudió tres formas de

\subsection{Variación en el contenido de vacíos}

El contenido de vacíos es un factor determinante en la variabilidad de los resultados del ensayo. Se ensayaron varios rangos de vacíos y los resultados obtenidos se presentan en la tabla 5 . Se puede observar que los resultados son más repetibles en el rango de vacíos entre $6,5 \%$ y $7,5 \%$. De acuerdo con lo anterior, se puede establecer un rango de $(7,0 \pm 0,5) \%$ pero esto resulta en la realización de muchos especímenes por lo que el rango establecido de $(7 \pm 1) \%$ está bien. 


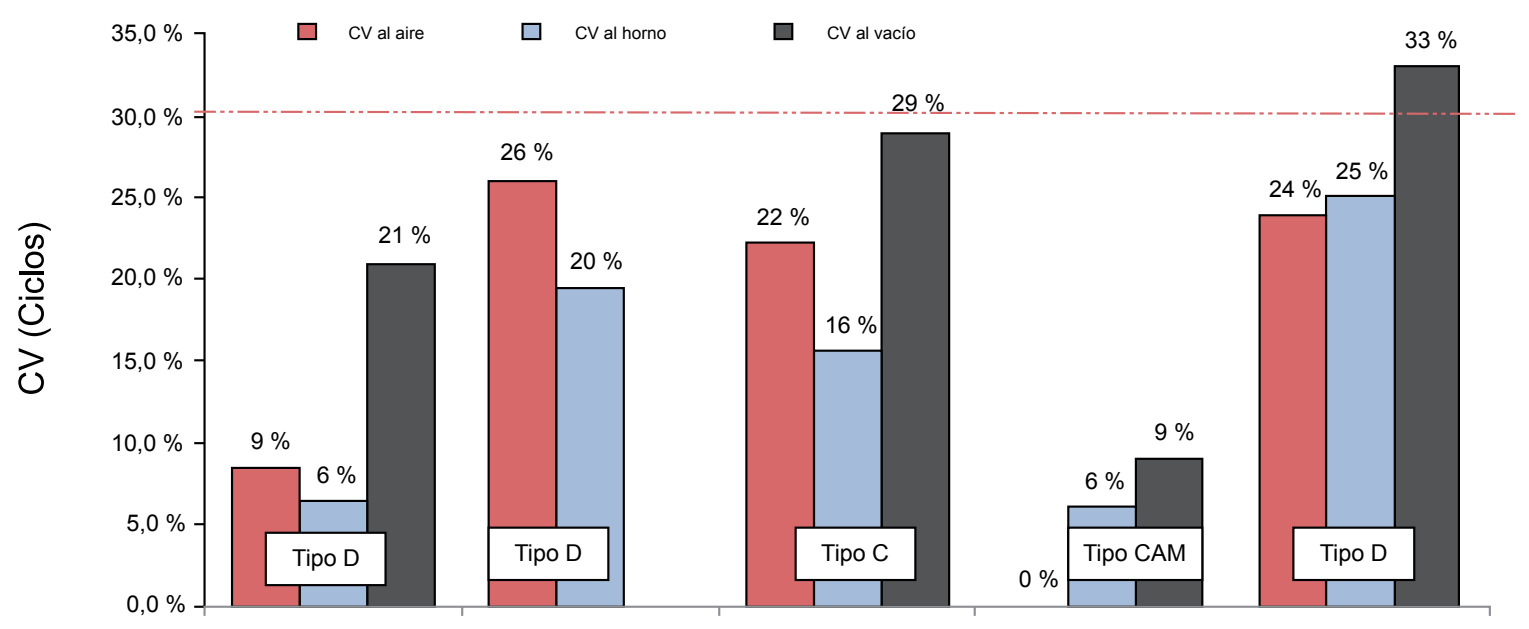

Figura 16. Comparación de los métodos de secado

Fuente: Walubita et al, Fgura H-1, 2012 [Ref. 5]

Tabla 5. Resultados para los distintos rangos de vacíos. Fuente: Walubita et al. Tabla 5-3. 2012 [Ref. 5]

\begin{tabular}{|c|c|c|c|c|c|c|}
\hline \multirow{2}{*}{$\begin{array}{c}\text { Rango de } \\
\text { vacíos (\%) }\end{array}$} & \multicolumn{2}{|c|}{ Chico Tipo D } & \multicolumn{2}{|c|}{ Atlanta Tipo D } & \multicolumn{2}{c|}{ Laredo Tipo C } \\
\cline { 2 - 7 } & Promedio & CV & Promedio & CV & Promedio & CV \\
\hline $5,0-5,5$ & -- & -- & 83 & $66 \%$ & -- & -- \\
\hline $5,5-6,0$ & -- & -- & 176 & $0 \%$ & 55 & $29 \%$ \\
\hline $6,0-6,5$ & 89 & $11 \%$ & 187 & $72 \%$ & 49 & $13 \%$ \\
\hline $6,5-7,0$ & 254 & $6 \%$ & 116 & $6 \%$ & 92 & $8 \%$ \\
\hline $7,0-7,5$ & 80 & $9 \%$ & 144 & $11 \%$ & 53 & $23 \%$ \\
\hline $7,5-8,0$ & 162 & $44 \%$ & 188 & $25 \%$ & -- & -- \\
\hline $8,0-8,5$ & 165 & $60 \%$ & -- & -- & -- & -- \\
\hline
\end{tabular}

\subsection{Tipo de epóxico, cantidad y criterio de pegado}

En el método estándar Tex-248-F, se establece el uso de un epóxico de dos fases pero no indica cuánta cantidad se requiere, es por esto que estudiaron 3 cantidades: $14 \mathrm{~g}$, $16 \mathrm{~g}$ y $18 \mathrm{~g}$. Los $14 \mathrm{~g}$ es una cantidad insuficiente mientras que $18 \mathrm{~g}$ resultó en un exceso provocando mucho derrame, por lo tanto, $16 \mathrm{~g}$ es una cantidad suficiente para obtener resultados repetibles.

En cuanto al tipo de epóxico, se descarta el uso del epóxico Devcon Plastic Steel Putty pues el tiempo de secado es extenso pues se requiere más material para recubrir toda el área y el costo del mismo es alto y es poco trabajable. Los autores recomiendan el uso del epóxico de dos fases con una resistencia mínima de 17,2 MPa (2 500 psi).

\subsection{Variación en la temperatura de ensayo}

En general, cuando se aumenta la temperatura de ensayo se aumenta la cantidad de ciclos de falla, esto debido a que el asfalto se hace menos rígido y la mezcla se hace más dúctil. Se hicieron pruebas a distintas temperaturas para dos tipos de mezcla y se llegó a la conclusión que la temperatura de ensayo $(25,0 \pm 0,5)^{\circ} \mathrm{C}$ está bien y que si la temperatura cambia en más de $2{ }^{\circ} \mathrm{C}$ ya la diferencia en los resultados es significativa.

\subsection{Parámetros de carga y periodo de relajación del ensayo}

Se estudió cambiar la distancia de abertura de carga, se realizaron ensayos reduciendo la distancia de $0,635 \mathrm{~mm}$ a $0,51 \mathrm{~mm}$ y $0,38 \mathrm{~mm}$. Se concluyó que reducir esta distancia mejoró el desempeño, pero sin cambios en la carga máxima o variabilidad, por lo que recomiendan que esto no se cambie y se mantenga la frecuencia de carga en 10 segundos/ciclo.

Cuando se monta el espécimen en el equipo se debe esperar que se alcance el equilibrio en la temperatura pero también se tiene que la muestra se recupere elásticamente luego de que los tornillos son socados pues esto puede tener efectos negativos en los resultados. Se estableció que se tiene que dejar 10 minutos como mínimo de tiempo de relajación.

\subsection{Abertura entre placas}

Se estudió el uso de nuevas placas base y el uso de una barra metálica para mantener la distancia de $2 \mathrm{~mm}$ pero resultó complicado quitar la barra a la hora de poner el epóxico y la muestra. 


\section{Conclusiones y recomendaciones}

El ensayo de Overlay es un método efectivo y rápido para determinar la susceptibilidad al agrietamiento de mezclas asfálticas a nivel de laboratorio, pues simula el mecanismo de apertura y cierre de las grietas o juntas, sin embargo, la repetibilidad y variabilidad de los resultados son factores a considerar. La implementación del ensayo en el LanammeUCR fue exitosa.

La preparación de los especímenes requiere de mucho cuidado pues afecta de manera considerable los resultados, por lo que es mejor compactar un espécimen para sacar dos briquetas para minimizar el desperdicio. Es mejor ensayar 5 briquetas mínimo para luego promediar los 3 resultados que presenten la menor variabilidad, en el proceso de implementación del ensayo se decidió seguir preparando 6 briquetas pues esto significa compactar 3 especímenes de $127 \mathrm{~mm}$ de altura en el Compactador Giratorio Superpave ${ }^{\circledR}$.

Actualmente, el método Tex-248-F indica que las briquetas se pueden secar a una temperatura de $(60 \pm 3){ }^{\circ} \mathrm{C}$, pero esta temperatura es demasiado alta por lo que se cambió a un rango de $(40 \pm 3)^{\circ} \mathrm{C}$ durante 12 horas y es mejor que el secado al aire.

El contenido de vacíos de aire debe estar en el rango de $(7 \pm 1) \%$, pero este rango corresponde a los vacíos de la briqueta por lo que a la hora de compactar los especímenes estos deben tener un contenido mayor de vacíos pues cuando se cortan este valor tiende a disminuir. Para las pruebas realizadas para la implementación se compactaron los especímenes a un contenido de vacíos de 8,5\%. Este es un proceso que se tiene que realizar a prueba y error lo que significa mayor tiempo de ensayo.

Las briquetas se tienen que ensayar preferiblemente en 5 días máximo después de su preparación, por lo que esto se tiene que tomar en cuenta a la hora de preparar los especímenes de ensayo y también tomar en cuenta la duración del ensayo.

La escogencia del epóxico y la cantidad a utilizar es de suma importancia, es por esto que se utilizó el epóxico de dos fases Epoxibonder transparente para el montaje de las briquetas. Lo que se hizo fue colocar cinta adhesiva en el centro de la cara que se va a pegar a las placas, y se pesó 8 gramos para un lado y 8 gramos en el otro lado y se procedió a mezclar el epóxico sobre estas caras para evitar el desperdicio y que no se coloque la misma cantidad en ambos lados. Luego se retiró la cinta adhesiva y se pegó la briqueta a las placas.

La duración de un ensayo puede ser de 1 hora hasta 3 horas, esto dependerá de las características de la mezcla asfáltica, para los ensayos de implementación se utilizó una muestra sin modificar y otra modificada con látex lo que permitió que se observara la mejoría del uso del aditivo en la mezcla, lo que indica que este ensayo puede de manera efectiva diferenciar la resistencia al agrietamiento entre distintas mezclas asfálticas.

\section{Referencias}

1. Tex-248-F (2009). Overlay Test. Accesado en setiembre de 2012. http://www.google.co.cr/url?sa $=t \& r c t=j \& q=\& e s r c=s \&$ source $=$ web\&cd $=2 \& v e d=0 C D U Q F j A B \& u r l=h t t p \% 3 A \% 2 F \% 2 F f t p . d o t$. state.tx.us\%2Fpub\%2Ftxdot-info\%2Fcst\%2FTMS\%2F200-F_ series\%2Fpdfs\%2Fbit248.pdf\&ei=K1sJUqLwM9XA4APGloDwBw \&usg=AFQjCNHebjNf49QJlgo7JbQ_LJzP8Q1eSw\&sig2=KQ_Jc_ Gyp3v3vycsr9yQFw\&bvm=bv.50500085, d.dmg

2. Loría Salazar, L. (2008). Reflective Cracking of Flexible Pavements: Literature Review, Analysis Models, and Testing Methods. Tesis de maestría, Universidad de Nevada, Reno. Estados Unidos.

3. Zhou, F. and Scullion, T. (2005). Overlay Tester: A rapid performance related crack resistance test. Reporte de investigación FHWA/TX05/0-4467-2, Texas Transportation Institute, Texas A\&M University System, College Station, Texas. Estados Unidos.

4. Zhou, F. and Scullion, T. (2003). Upgraded Overlay Tester and its application to characterize reflection cracking resistante of asphalt mixtures. Reporte de investigación FHWA/TX-04/0-4467-1, Texas Transportation Institute, Texas A\&M University System, College Station, Texas. Estados Unidos.

5. Walubita, L., Faruk, A., Das, G., Tanvir, H., Zhang, J., y Scullion, T. (2012). The Overlay Tester: A sensitivity study to improve repeatability and minimize variability in the test results. Reporte de investigación No. FHWA/TX-12/0-6607-1, Texas Transportation Institute, Texas A\&M University System, College Station, Texas. Estados Unidos.

6. Washington State Department of Transportation. (2008). Texas Overlay Tester Project. Nota técnica Laboratorio de Materiales, Washington State. Estados Unidos.

7. IPC Global. (2012). Overlay Tester Accessories - AMPT/UTM. AMPT OLT0512. Boronia, Australia. 